\title{
Energy Optimization for Underwater Sensor Network using Nature Inspired Technique
}

\author{
Sukhman Kaur
}

\begin{abstract}
There is lots of energy optimization technique is used in underwater sensor network but in this paper, nature-inspired technique, called Elephant Herding Optimization (EHO), and is proposed for solving optimization tasks. The EHO method is inspired by the herding behavior of the elephant group. In nature, the elephants having a place with various factions live respectively under the initiative of female authority, and the male elephants will leave their family bunch when they grow up. These two practices can be displayed into two after administrators: group refreshing administrator and isolating administrator. In $\mathrm{EHO}$, the elephants in every faction are refreshed by its present position and female authority through group refreshing administrator. It is found from the outcomes that the EHO based vitality streamlining approach shows the outcomes compelling than established methodology as far as numerous parameters. In this work, the advancement dimension of the vitality to over $11 \%$ is accomplished utilizing Elephant Herd Optimization that is likewise utilized in numerous other areas for designing enhancement.
\end{abstract}

Index Terms: Energy Optimization Underwater Sensor Networks, Elephants, Nature Inspired Techniques.

\section{INTRODUCTION}

There are many numbers of nature aspired algorithms for energy optimizations. Moreover, though modern nature aspired approaches cannot provide exact answers; they can generate satisfactory solutions within a reasonable time span. Over the past few years, various kinds of nature aspired girths have been proposed and successfully applied to solve myriads of real-world optimization problems. Among all nature aspired methods, swarmbased algorithms are one of the most representative paradigms \& widely used ones. Its inspiration originates from the collective behavior of animals. The ants, in nature, are well capable of keeping the past paths in mind by pheromone. More effective swarm intelligence algorithms have been proposed, such as artificial bee colony (ABC) [4][5], Recently, Inspired by this phenomenon, ACO [10][11] was proposed by Dorigo et al. cuckoo search (CS) [9-12],Moreover, firefly algorithm (FA) [22], ant lion optimizer (ALO) [25] ,bat algorithm (BA) [26[27] , big bang-big crunch algorithm (BB-BC) [29], charged system search (CSS) [29-32], multi-verse optimizer (MVO)[33] ,krill herd (KH)[3437], chaotic swarming of particles (CSP) [38], monarch butterfly optimization (MBO) [40], and many more.

In modern era, A number of algorithms and approaches including nature inspired algorithms are developed and implemented to cope up the issues of energy optimization still in this paper aspects of Elephant Herd Optimization (EHO) is used to improved it to be followed by various sensor nodes with the help of cluster Head.

\section{Revised Manuscript Received on July 02, 2019.}

Sukhman kaur, Department of Computer Science, Punjabi University, Patiala, India.
The wireless nodes with degree of energy and lifetime shall be given occasion to be cluster head so that the result of the clustered environment can be escalated. Using this technique performance of the selecting of nodes using cluster head is more improved it takes less time.

\section{Elephant Herd Optimization (EHO) for Energy optimization in UWSN}

By and large, wide elephants are social in nature and the elephant bunch is made out of a few factions. The elephants having a place with various families live respectively under the authority of a matron, and male elephants stay singular and will leave their family gathering while at the same time growing up. Enlivened by the crowding conduct of elephant gathering, another sort of swarm-based heuristic hunt technique, called EHO, is proposed for explaining worldwide advancement assignments. This residence of elephants can be utilized to take care of advancement issues. The conduct of elephant grouping in nature is glorified into family refreshing administrators and isolating administrator. In EHO, each elephant actualizes tribe refreshing administrator to refresh its position dependent on its present position and female authority in the reacting group. Therefore, the most exceedingly terrible elephant is supplanted by isolating the administrator. By contrasting and $\mathrm{BBO}, \mathrm{DE}$ and GA, the execution of EHO are explored by a few examinations executed on fifteen experiments. The results demonstrate that $\mathrm{EHO}$ can discover a lot of fitter arrangements on most benchmark issues than the three different techniques.

The issue definition and research holes while recognizable proof of the issue is that the vitality advancement is a significant enormous area that is under research and still gigantic vitality misfortune situations happen in the submerged sensor systems correspondence. The prior is having grouped needs in the multilayered and multi- dimensional model for vitality enhancement and The methodology of Elephant Herd Optimization(EHO)

\section{FLOW OFWORK}

1) Analysis of the traditional theories and algorithms for energy optimization in underwater sensornodes.

2) Assessment of the energy levels in wireless motes at the initial level with the goal that the last qualities can think about after enhancement calculation.

3) Choosing the fitting simulation tool/implementation software. In this work, the implementation is done in MATLAB.

4) Generation of the motes in varying numbers so that different scenarios in the

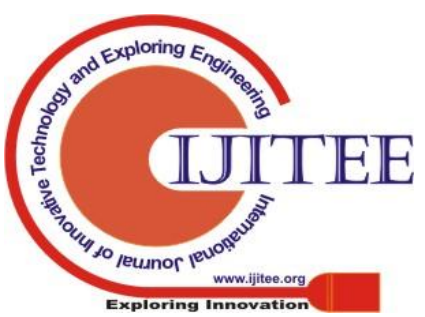


deployment area can be investigated.

5) Setup for implementation of Existing / Classical and Proposed(EHO).

a) Cluster Head Formation Process using Classical and EHO Initiates.

6) Implementation of Elephant Herd Optimization (EHO) by treating the sensor nodes as elephants.

a) Consideration of Sensor Nodes as Elephants.

b) Each Sensor Node having specific Energy and Lifetime.

c) EHO based dynamic cluster head formation.

7) Choosing the best wireless node as Most Fit Cluster Head as Female Elephant Strategy in EHO.

8) Keeping track of the energy levels and consumption parameters.

9) Execution of the simulation scenario in different nodes and final evaluation of the outcome.

10) Detailed Logs Generation and Analysis of different Parameters:
a) Execution Time
b) Cost Factor
c) Complexity
d) Performance /Efficiency

Algorithm Elephant Herding Optimization (EHO)

1) Initialization. Age of Individuals; Division of Elephant Population to $\mathrm{n}$ number of Groups; Computation of the Fitness Score with Each Individual; initialize the Counter $\mathrm{i}=1$ and $\max$ population in currentGroup

2) while i <MaxPopulationdo

a) Arrange solutions according to fitness core

3) for all groups gido

4) for all solutions $m$ in the group gdo

a) Updatexgi, $\mathrm{m}$ and generate $\mathrm{x}_{\text {new }}, \mathrm{g}_{\mathrm{i}}$, musing FuzzyFitness

b) Select and Use better solution between $\mathrm{Xgi}, \mathrm{m}$ and $\mathrm{x}_{\text {new }}, \mathrm{g}_{\mathrm{i}}, \mathrm{m}$

c) Update xbest and generation of new, $\mathrm{g}_{\mathrm{i}}, \mathrm{m}$ using fitnessscore

d) Select the best solution and set inxbest

5) endfor

6) for all group $\mathrm{g}_{\mathrm{i}}$ in the population-setdo

a) Replacement of the worst solution

in the group-clan $\mathrm{g}_{\mathrm{i}}$ using

currentscore

7) endfor

a) Evaluation of population and calculation of fitness

8) endwhile

9) return the best-fit solution xbest in thegroup-clans

10) If the optimal solution achieved. Stop else go to step 3End

\section{IMPLEMENTATION RESULTS AND OUTCOME}

To actualize the energy optimization methodology utilizing Elephant Herd Optimization, the recreation of the remote condition is done in MATLAB utilizing the Biography tool compartment to delineate diverse sensor hubs. The following are the screen captures and yield acquired from the recreation situations in various time ranges.

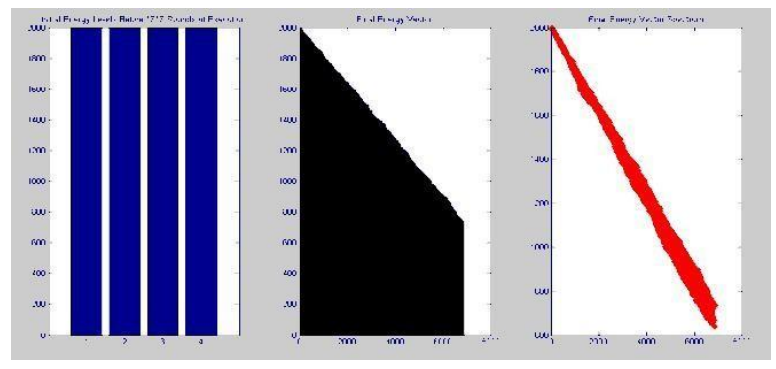

Fig. (a) ANALYSIS OF ENERGY LEVELS AND ALIVE NODES

Fig (a) presents the analysis of Energy Levels and Alive Nodes. In the traditional base work, the graph is mentioned with the ending of alive nodes at around 2500 rounds. This overall process is optimized for a higher degree of effectiveness.

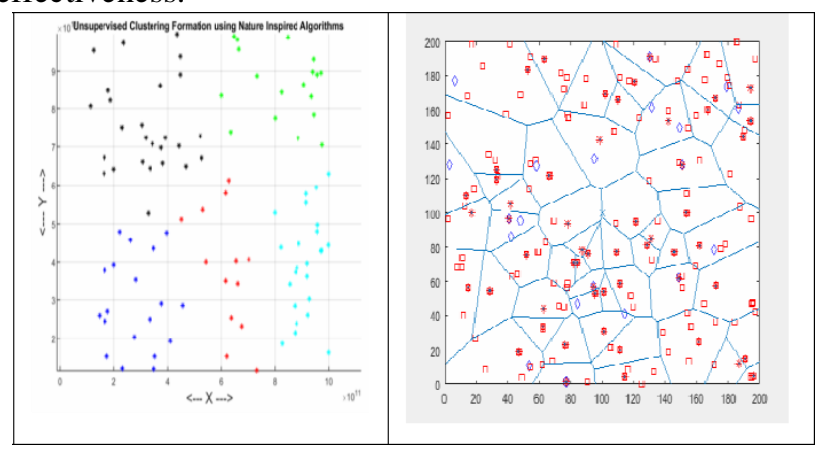

Fig. (b) cluster formation in wireless environment

Fig (b) depicts the scenario of Cluster Formation in the wireless environment and it is visible in the results that multiple nodes are in the grouping phase for the data transmission and overall energy optimization. 


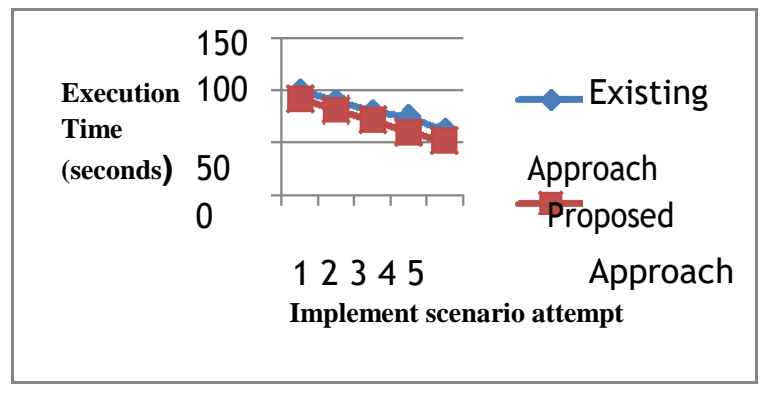

Fig. (c) EVALUATION OF EXECUTION TIME

Fig (c) presents the comparison of execution Time in the existing (Ant colony) and proposed EHO approach. It is evident from the results that the execution time of EHO is comparatively less than the existing approach.

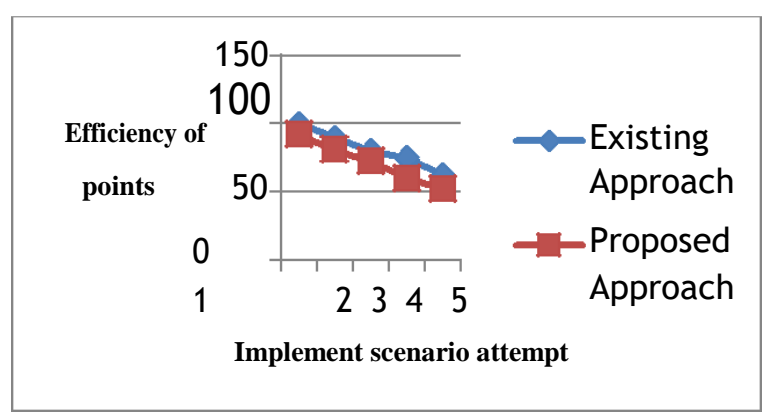

Fig.(d) EVALUATION OFEFFICIENCY

Fig (d) depicts that the efficiency of the proposed EHO approach is much better from the existing approach.

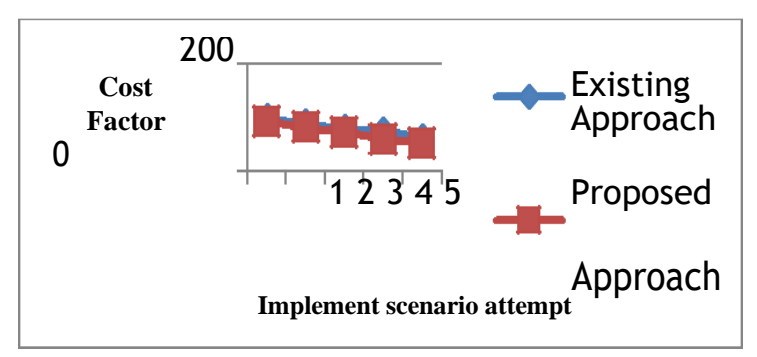

Fig. (e) THE SIMULATION RESULTS IN EXISTING AND PROPOSED APPROACH IN TERMS OF COST.

Fig (e) presents the evaluation and comparison of the cost factor in the traditional and the novel approach of elephant herd optimization (EHO). The proposed approach is presenting the results effective in terms of less cost factor as compared to the existing approach of the Ant Colony approach.

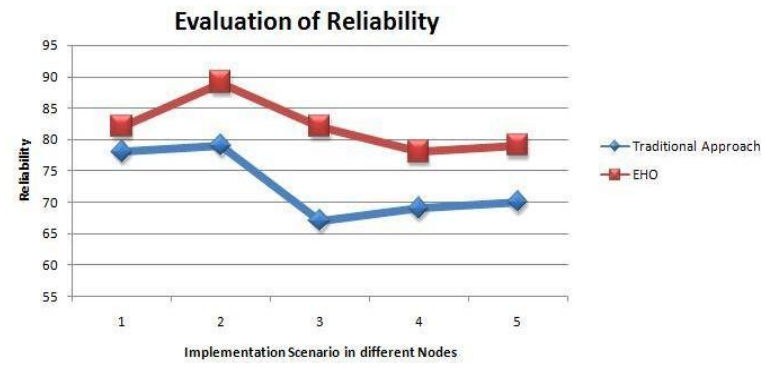

Fig. (f) EVALUATION OF RELIABILITY
Figure (f) presents the reliability and related perspectives in the traditional and proposed EHO approach. Traditional Approach is not having more reliability as compared to the EHO based approach. The Traditional is taken as the implementation without the integration of met heuristic based simulation. It is evident from the results that this factor of EHO is comparatively effective than the traditional approach.

\section{CONCLUSION}

Nature Inspired Approaches are widely used for solving optimization problems from a long time and that's why this dimension is adapted to be implemented in the wireless networks. The proposed approach is evaluated on multiple parameters including energy optimized, accuracy, turnaround time and overall performance of the network. In this proposed algorithm, an efficient routing technique is meant to be followed by various sensor nodes with the help of Cluster Heads. In addition and for further improvements, nature-inspired approaches and soft computing approaches can be used to achieve global optimization. As deep Learning is one of the constituents of soft computing having core tasks associated with classification, a recognition which is generally related to artificial intelligence.

\section{REFERENCES}

[1] X.Gao,Z.Cui,,"Theoryandapplicationsofswarmintelligence," Neural Computing \& Applications, vol. 21.

[2] J. Kennedy, R. Eberhart, "Particle swarm optimization," in Proceeding of the IEEE International Conference onNeural Networks, Perth, Australia, 1995.

[3] S. Mirjalili, L. d. S. Coelh, G.-G.Wang, "Binary optimizationusing hybrid particle swarm optimization and gravitational search algorithm," Neural Computing and Applications, vol.25.

[4] A. H. Alavi, A. H. Gandomi, G.-G. Wang, S. Deb, "A hybrid method based on krill herd and quantum-behaved particle swarm optimization," Neural Computing and Applications, 2015.

[5] G.-G. Wang, A. H. Gandomi, X.-S.Yang, A. H. Alavi, "A novel improved accelerated particle swarm optimization algorithm for global numerical optimization," Engineering Computations, vol.31.

[6] X. Zhao, B. Song, P. Huang, Z. Wen, J. Weng, Y. Fan, X. Zhao, B. Song, "An improved discrete immune optimization algorithm based on PSOforQoS-drivenwebservicecomposition,"AppliedSoftComputing, vol.12.

[7] X.Zhao,"Aperturbedparticleswarmalgorithmfornumerical optimization," Applied Soft Computing, vol.10.

[8] S. Mirjalili, A. Lewis, "S-shaped versus V-shaped transfer functions for binary Particle Swarm Optimization," Swarm and Evolutionary Computation, vol.9.

[9] M. Dorigo, V. Maniezzo,A. Colorni, "Ant system: optimization by a colony of cooperating agents,"IEEETransactions on Systems,Man, and Cybernetics, Part B:Cybernetics, vol. 26.

[10] J. Jaen, K. Krynicki , J. A. Mocholí, "Ant colony optimization for resource searching in dynamic peer-to-peer grids," International Journal of Bio- Inspired Computation, 2014,vol. 6, no. 3, pp. 153-165.

[11]D.Karaboga,B.Basturk, “Apowerfulandefficientalgorithm for numerical function optimization: artificial bee colony (ABC) algorithm," Journal of Global Optimization,2007, vol. 39, no. 3, pp. 459-471.

[12]X. S. Yang, S. Deb, "Cuckoo search via Lévy flights." pp.210-214.

[13]S. Debv, X.-S. Yang, "Cuckoo search: recent advances and applications," Neural Computing and Applications,2013 vol. 24.

[14]X. Li, J. Wang, M. Yin, "Enhancing the performance of 


\section{Energy Optimization Approach For Underwater Sensor Network Using Nature Inspired Technique}

cuckoo search algorithm using orthogonal learning method,'NeuralComputing and Applications, 2013,vol. 24, no. 6.

[15]A. H. Gandomi, X. Zhao, G.-G. Wang, H. C. E. Chu,"Hybridizing harmony search algorithm with the cuckoo search for global numerical optimization," Soft Computing,2014.

[16] G.-G.Wang,S.Deb,A.H.Gandomi,Z.Zhang,A.H.Alavi, “Chaotic cuckoo search," Soft Computing, 2015.

[17] A. H. Gandomi, G.-G. Wang, A. H. Alavi, X.-S.Yang, “A new hybridmethodbasedonkrillherdandcuckoosearchforglobal optimization tasks," International Journal of Bio-Inspired Computation, 2012.

[18]X. Li, M. Yin, "Modified cuckoo search algorithm with selfadaptive parameter method," Information Sciences, 2015, vol. 298.

[19] X. S. Yang, "Nature-inspired metaheuristic algorithms", 2nded., Luniver Press, Frome,2010.

[20]S. M. Mirjalili, X.-S. Yang, "Binary bat algorithm,"Neural Computing and Applications, 2013,vol. 25.

[21]X.-S. Yang, A. H. Gandomi, A. H. Alavi, "Mixed variable structuraloptimizationusingfireflyalgorithm,"Computers\&Structure s, 2011, vol. 89.

[22]X.S.Yang,"Fireflyalgorithm,stochastictestfunctions, anddesign optimization," International Journal of Bio-Inspired Computation, 2010, vol. 2.

[23] G.-G. Wang, D. Wang, "An effective hybrid firefly algorithm with harmonysearchforglobalnumericaloptimization,"The ScientificWorld Journal, 2013.

[24] S.Mirjalili,"Theantlionoptimizer,"AdvancesinEngineering Software, 2015, vol. 83.

[25] J.-W. Zhang, G.-G. Wang, "Image matching using a bat algorithm with mutation," Applied Mechanics and Materials, 2012,vol. 203.

[26]B. Chang, G.-G. Wang, Z. Zhang, "A multi-swarm bat algorithm for globaloptimization."

[27] A.Kaveh,S.Talatahari,"Adiscretebigbang-bigcrunchalgorithmfor the optimal design of skeletal structures,",2010, .vol. 11.

[28]A. Kaveh, S. Talatahari, "Size optimization of space trusses using Big Bang-Big Crunch algorithm," Computers \& Structures, vol. 87,2009.

[29] S. Talatahari, "A novel heuristic optimization method:charged system search," Acta Mechanica, 2010, vol. 21.

[30]R. Sheikholeslami, S. Talatahari, "Optimum design of gravity and reinforced retaining walls using enhanced charged system search algorithm," KSCEJournalofCivilEngineering,2014,vol.18,no.5.

[31] S. Talatahari, A. Kaveh, "Charged system search for the optimal design of frame structures," Applied Soft Computing, 2012,vol. 12.

[32]A. Kaveh, R. Sheikholeslami, S. Talatahari, M. KeshvariIlkhichi, "Chaotic swarming of particles: a new method for size optimization of truss structures," Advances in Engineering Software,2014, vol. 67.

[33] S. M. Mirjalili, A. Hatamlou, "Multi-verse optimizer: a natureinspired algorithm for global optimization," Neural Computing and Applications, 2015.

[34]A. H. Gandomi, A. H. Alavi, "Krill herd: a new bio-inspired optimizationalgorithm,"CommunicationsinNonlinearScienceand Numerical Simulation, 2012,vol. 17, no. 12

[35]G.-G. Wang, A. H. Gandomi, A. H. Alavi, "A chaotic particleswarmkrill herd algorithm for global numerical optimization," Kybernetes, 2013,vol. 42.
[36] A. H. Gandomi, A. H. Alavi, G.-G. Wang, “An effective krill herd algorithm with migration operator in biogeography-based optimization," Applied Mathematical Modelling, 2014,vol. 38.

[37] A. H. Gandomi, G.-G. Wang, A. H. Alavi, "Stud krillherd algorithm," Neurocomputing, 2014,vol. 128.

[38] G.-G.Wang,A.H.Gandomi,A.H.Alavi,S.Deb, “AMulti-Stage Krill Herd Algorithm for Global Numerical Optimization,” International Journal on Artificial Intelligence Tools,2015.

[39] A.H.Alavi, G.-G.Wang,L.Guo,H.Duan,“'Anewimprovedkrillherd algorithm for global numerical optimization,"Neurocomputing, vol. 138, 2014.

[40] G.-G.Wang,S.Deb,Z.Cui,"Monarchbutterflyoptimization,” Neural Computing and Applications,2015.

\section{AUTHORS PROFILE}

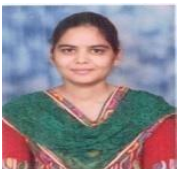

Sukhman Kaur received M.C.A degree in computer science and application from Punjabi University, Patiala, India, in 2015. She received an MPhil degree in computer science and application from Punjabi University, Patiala, India, in 2018. She is currently pursuing a Ph.D. degree in Computer Scienceat Punjabi University, Patiala, India. Her research interests include Data mining and networking. 